International Conference on Renewable Energies and Power Quality (ICREPQ'11)

Las Palmas de Gran Canaria (Spain), 13th to 15th April, 2011

\title{
Artificial Intelligence Techniques for Controlling Spacecraft Power System
}

\author{
Hanaa T. El-Madany ${ }^{1}$, Faten H. Fahmy ${ }^{1}$, Ninet M. A. El-Rahman ${ }^{1}$, and Hassen T. Dorrah ${ }^{2}$ \\ ${ }^{1}$ Photovoltaic cells Department \\ Electronics Research Institute \\ National Research Center Building Cairo, Egypt \\ E-Mail: hanaa tolba@yahoo.com \\ ${ }^{2}$ Electrical Power \& Machines Department \\ Cairo University \\ Egypt
}

\begin{abstract}
Advancements in the field of artificial intelligence (AI) made during this decade have forever changed the way we look at automating spacecraft subsystems including the electrical power system. AI have been used to solve complicated practical problems in various areas and are becoming more and more popular nowadays. In this paper, a mathematical modeling and MATLAB-SIMULINK model for the different components of the spacecraft power system is presented. Also, a control system, which includes either the Neural Network Controller (NNC) or the Fuzzy Logic Controller (FLC) is developed for achieving the coordination between the components of spacecraft power system as well as control the energy flows. The performance of the spacecraft power system is evaluated by comparing two control systems using the NNC and the FLC.
\end{abstract}

\section{Key words}

Spacecraft, Neural network, Fuzzy logic control, Photovoltaic array.

\section{Introduction}

Provision of electrical power for space vehicles is the most fundamental requirement for the satellite payload. Power system failure necessarily results in the loss of a space mission, and it is interesting to note that, many of the early satellite systems failed due to such a loss [1].

In the last three decades, numerous alternative control techniques, such as neural and fuzzy control, have been proposed instead of conventional classical technique. Development of artificial neural networks (ANN's) and fuzzy logic theory have inspired new resources for possible implementation of better and more efficient control. ANN's have capability of learning the dynamical systems that estimated input-output functions. Fuzzy systems transform sets of structured information into the appropriate control actions. Especially, neither ANN's nor fuzzy systems need mathematical modeling of the plants. Fuzzy control systems can be developed along with linguistic lines and need some expertise information about the plant. On the other hand, before used for control purposes, ANN's have to be trained and they need some information (not based on mathematical model but sometimes taken measurement from plant) about the plant. Generally, input-output characterization or desired output of the plant or neuro controller are sufficient $[2,3]$.

The emphasis of this paper is concerned with the control of the energy flow of spacecraft power system using either the NN or the FL techniques. The performance of the global system has been studied using MATLAB SIMULINK.

\section{Spacecraft Power System}

Photovoltaic conversion of the sun's energy is the most common source of electrical power in space. A typical solar panel-battery power system is shown in Fig. 1 [4, 5].

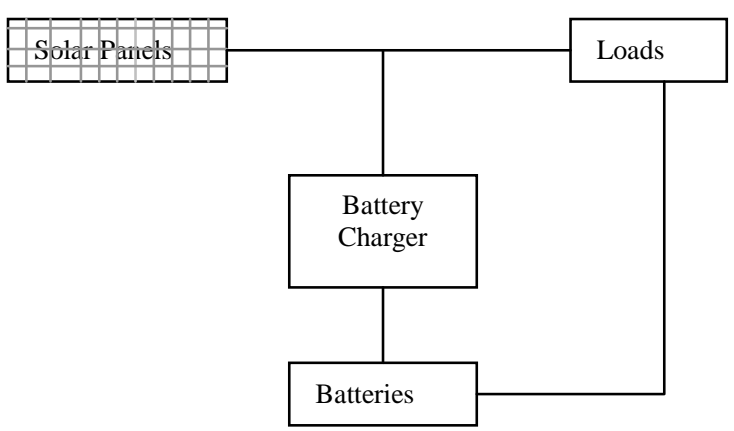

Fig. 1. Typical solar panel- battery system architecture. 


\section{PV Panel Model}

A photovoltaic cell is basically a $\mathrm{p}-\mathrm{n}$ semiconductor junction diode which converts solar light energy into electricity. Using the equivalent circuit of a solar cell, the non-linear $I-V$ characteristics of a solar array are extracted, neglecting the series resistance [6]:

$$
I_{o}=I_{p h}-I_{r s}\left(e^{q V_{o} / k T A}-1\right)-\frac{V_{o}}{R_{s h}}
$$

Where: $I_{0}$ is the PV array output current (A), $V_{0}$ is the PV array output voltage $(\mathrm{V}), q$ is the charge of an electron, $k$ is the Boltzmann's constant in $J / K, A$ is the $\mathrm{p}-\mathrm{n}$ junction ideality factor, $T$ is the cell temperature $(K)$, and $I_{\mathrm{rs}}$ is the cell reverse saturation current (A).

The photocurrent $I_{\mathrm{ph}}$ depends on the solar radiation and the cell temperature as described in the following equation:

$$
I_{p h}=\left(I_{s c r}+k_{i}\left(T-T_{r}\right)\right) \frac{s}{1353}
$$

Where: $I_{\text {scr }}$ is the PV array short circuit current (A) at reference temperature and radiation, $T_{\mathrm{r}}$ is the cell reference temperature, $k_{i}$ the short circuit current temperature coefficient $(A / K)$ and $S$ is the solar radiation $\left(\mathrm{W} / \mathrm{m}^{2}\right)$

\section{Artificial Intelligence Techniques}

\section{A. Fuzzy Logic Controller}

Fuzzy controllers have been proposed in diverse fields. The structure of the Fuzzy Controller (FC) is shown in Fig. 2. A fuzzy controller consists of a knowledge base, which contains the typical set of rules and an inference mechanism [6] and [7].

A fuzzification converts each piece of input data to degrees of membership by a lookup in one or several membership functions. The fuzzification block thus matches the input data with the conditions of the rules to determine how well the condition of each rule matches that particular input instance. There is a degree of membership for each linguistic term that applies to that input variable.

The rules may use several variables both in the condition and the conclusion of the rules. The controllers can therefore be applied to both multi-input-multi-output (MIMO) problems and single-input-single-output (SISO) problems.

The inference engine defines mapping from input fuzzy sets into output fuzzy sets. It determines the degree to which the antecedent is satisfied for each rule. The defuzzifier maps output fuzzy sets into a crisp number. Given a fuzzy set that encompasses a range of output values, the defuzzifier returns one number, thereby moving from a fuzzy set to a crisp number.

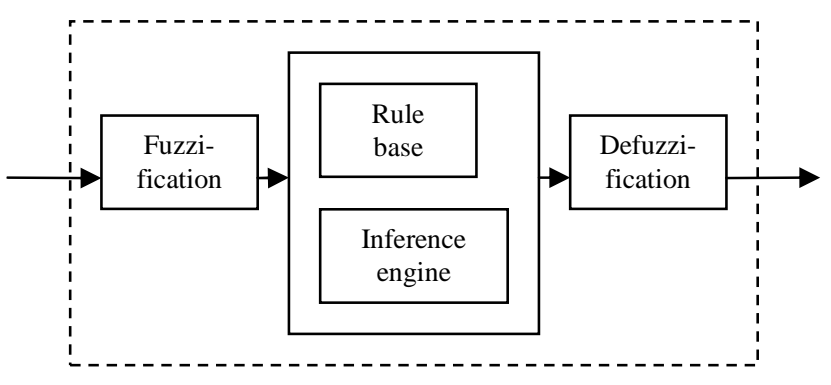

Fig. 2. General structure of fuzzy controller.

Benefits of fuzzy Logic

1) No need for a mathematical model.

2) Relatively simple, fast and adaptive.

3) Less sensitive to system fluctuations.

4) Can implement design objectives, difficult to express mathematically in linguistic or descriptive rules.

5) Based on intuition and judgment.

\section{B. Artificial Neural Network}

Artificial intelligence (AI) techniques are becoming useful as alternate approaches to conventional techniques or as components of integrated systems. They have been used to solve complicated practical problems in various areas and are becoming more and more popular nowadays. Nowadays, considerable attention has been focused on use of ANN on system modeling and control applications [8].

The basic processing elements of neural networks are called artificial neurons, or simply neurons or nodes. As indicated in Fig. 3, the effects of the synapses are represented by connection weights that modulate the effect of the associated input signals, and the nonlinear characteristic exhibited by neurons is represented by a transfer function [9]. The neuron impulse is then computed as the weighted sum of the input signals, transformed by the transfer function. The learning capability of an artificial neuron is achieved by adjusting the weights in accordance to the chosen learning algorithm. The learning situations in neural networks may be classified into three distinct sorts. These are supervised learning, unsupervised learning, and reinforcement learning [10] and [11].

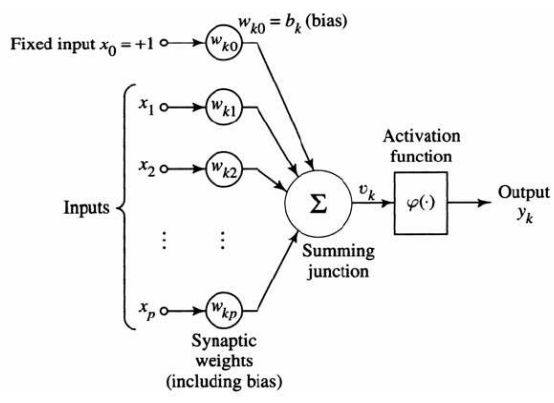

Fig. 3. Nonlinear model of a neuron. 
The main advantages of the neural network technique are:-

1) Nonlinearity.

2) Mapping input signals to desired response.

3) Adaptivity.

4) Evidential response: confidence level improves classification.

5) Contextual information: Knowledge is represented by the very structure and activation.

6) Fault tolerent: graceful degradation of performance if damaged.

7) Uniformity of analysis and design.

8) Neurobiological analogy

\section{Control Strategy}

\section{A. Proposed fuzzy logic controller}

Traditional FLC requires the expert knowledge of the process operation for the FLC parameter setting, and the controller can be only as good as the expertise involved in the design [12] and [13]. Fig. 4 indicates the proposed architecture of FLC of spacecraft power system. In this diagram, the FLC controls whether the system is in peak power or in eclipse conditions.

The inputs to the FLC are: (a) the error signal (e), indicating the difference between the output generation and the reference load, and (b) the derivative of this error signal (ce). The output of the FLC is the change in battery charge current $\left(\Delta I_{B C}\right)$. Standard triangular membership functions, as shown in Fig. 5 , are chosen for both the inputs and output of the fuzzy logic controller where NB, NM, NS, ZE, PS, PM, and PB are negative big, negative medium, negative small, zero, positive small, positive medium and positive big respectively. The fuzzy rule table of these sets is given in Table 1.

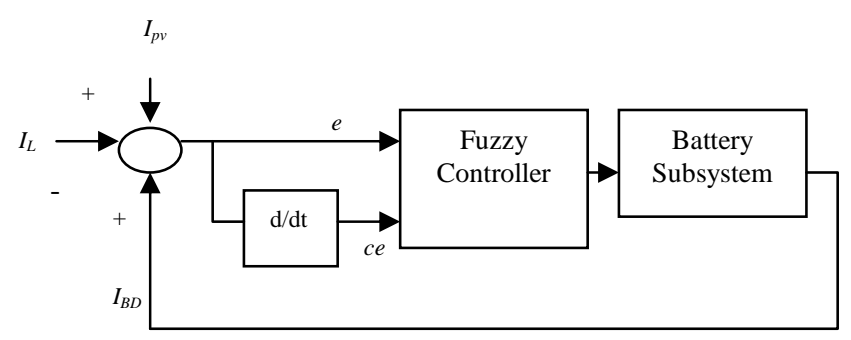

Fig. 4. Block diagram of proposed FLC.

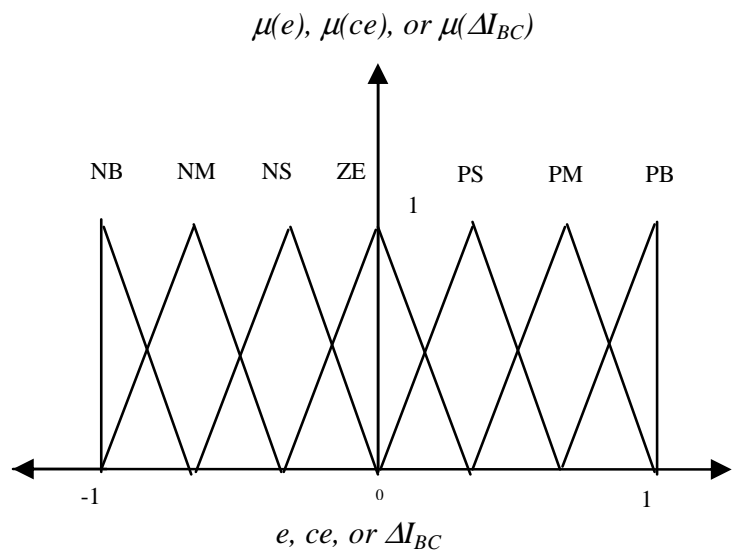

Fig. 5. Membership functions of the fuzzy sets.
Table 1: The rule table of the designed FLC.

\begin{tabular}{|c|c|c|c|c|c|c|c|c|}
\hline \multicolumn{2}{|c|}{$\Delta I_{B C}$} & \multicolumn{7}{|c|}{ Change in Error (ce) } \\
\cline { 2 - 9 } & NB & NM & NS & ZE & PS & PM & PB \\
\hline \multirow{2}{*}{} & NB & NB & NB & NB & NB & NM & NS & ZE \\
\cline { 2 - 9 } & NM & NB & NB & NB & NM & NS & ZE & PS \\
\cline { 2 - 9 } & NS & NB & NB & NM & NS & ZE & PS & PM \\
& ZE & NB & NM & NS & ZE & PS & PM & PB \\
\cline { 2 - 8 } & PS & NM & NS & ZE & PS & PM & PB & PB \\
\cline { 2 - 9 } & PM & NS & ZE & PS & PM & PB & PB & PB \\
\cline { 2 - 9 } & PB & ZE & PS & PM & PB & PB & PB & PB
\end{tabular}

\section{B. Proposed Neural Network Controller}

The power control unit controls whether the system is in peak power or in eclipse conditions comparing the solar array current with the load current, the difference between them is the change in battery charge current. An advantage of the neural network control over traditional control is its self-learning ability. As a result, so extensive prior information about the system is not required. Off line training for the proposed NNC was applied. Data for off-line training can be obtained either by simulation or experiment. The network is trained to recognize the relationships between the input and output parameters.

Back propagation algorithm is chosen which is a form of supervised learning for multi-layer nets. In this technique, the interlayer connection weights and the processing elements' thresholds are first initialized to small random values. The network is then presented with a set of training patterns, each consisting of an example of the problem to be solved (the input) and the desired solution to this problem (the output). These training patterns are presented repeatedly to the ANN model and the adjustment is performed after each iteration whenever the network's computed output is different from the desired output. This process continues until weights converge the desired error level or the output reaches an acceptable level. For this present work, the data is obtained by simulating the proposed system. After many trials, the developed NNC, shown in Fig. 6 eventually employed a 2-neuron input, a 3-neuron hidden layer, and one neuron output layer. The input network parameters are; the load current and the error signal while the output is the change in battery charge current $\left(\Delta I_{B C}\right)$. 


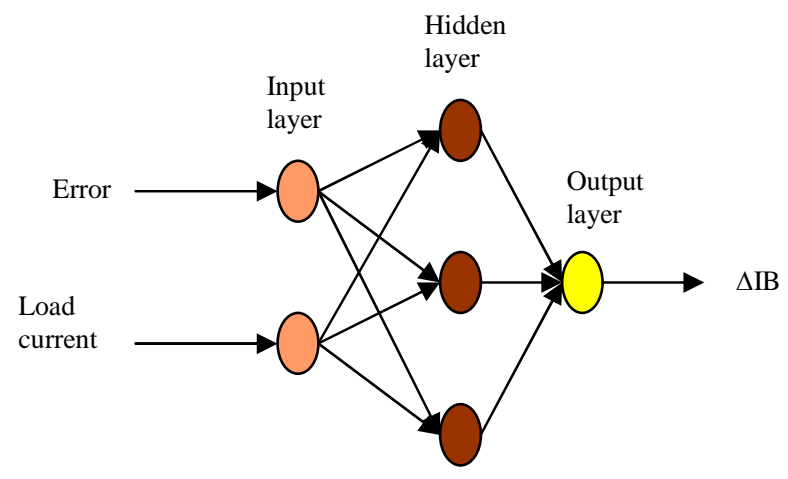

Fig. 6. The architecture of the NN controller model.

\section{Matlab Simulation Results}

In this section, models of the basic components of the suggested system are simulated using Matlab Software. These are the PV array, the battery storage and the control subsystems. A control system, which includes the NNC or the FLC controller is developed for achieving the coordination between the components of stand alone spacecraft power system as well as control the energy flows. The simulation block diagrams of spacecraft power system using FLC are shown in Fig. 7.

The inputs to The PV subsystem are insolation and temperature variables for one orbital period. While, the outputs are the PV current and power. The battery component as shown has one input, the charge current because the temperature is assumed to be constant during the operation as a result of its isolation from the space environment.

Fig. 8 depicts the simulink block diagram of FLC. The proposed FLC multi input single output (MISO) has twoinput single-output. The controller inputs are the error (e) and the change of error (ce) at the current sampling interval $\mathrm{k}$, while the controller output is the change in battery charge current $\left(\Delta I_{B C}\right)$.

Fig. 9 indicates the second proposed control technique using NNC. The input and the output are fixed initially however the number of hidden layers and the neurons within these layers are optimized during the learning process based on the good performance of root mean square error (RMSE). A two layer feed-forward network with "logsigmoid" hidden neurons and "purlinear" output neurons is be used. The network will be trained with Levenberg-Marquardt back propagation algorithm.

The solar insolation and the temperature profile in LEO indicated in Fig. 10 [14]. The typical current behavior of the PV array system is shown in Fig. 11. It is indicated that the variations of PV current follows the variations of the sun intensity. There will be time periods when the PV system is unable to meet the load demand (eclipse period). This implies that the PV system will need a storage system that will be able to provide enough energy during such period.

Using FLC and NNC, the PV output power, the battery power, and the load power profile are shown in Fig. 12 \&
13 respectively. It is clear that during sun periods, the generated power from PV feeds the load and the excess power charges the battery. In the contrary, during eclipse periods, the PV array unable to supply the load demand so the battery feeds the spacecraft subsystems. The positive values of the battery power refer to the charge mode. In the contrary, the negative values indicate the discharge mode.

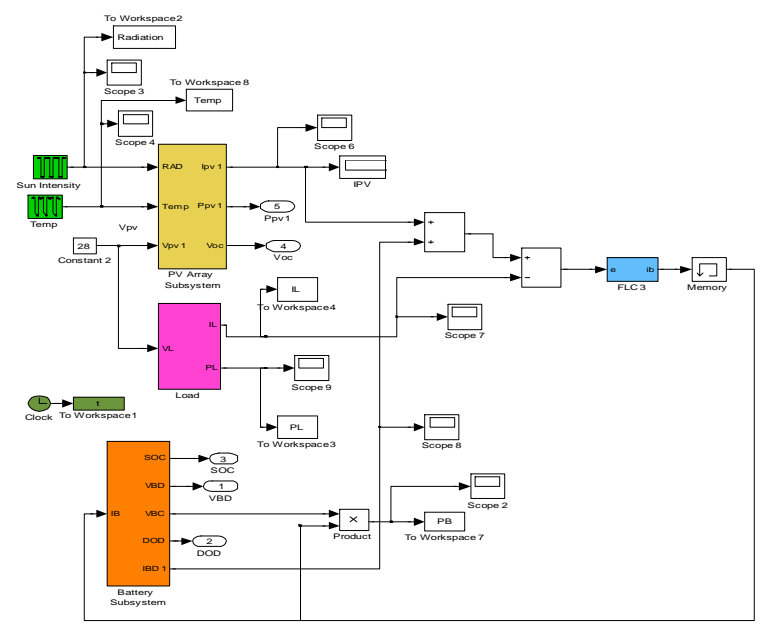

Fig.7. Simulink block diagram of the spacecraft power system with FLC.

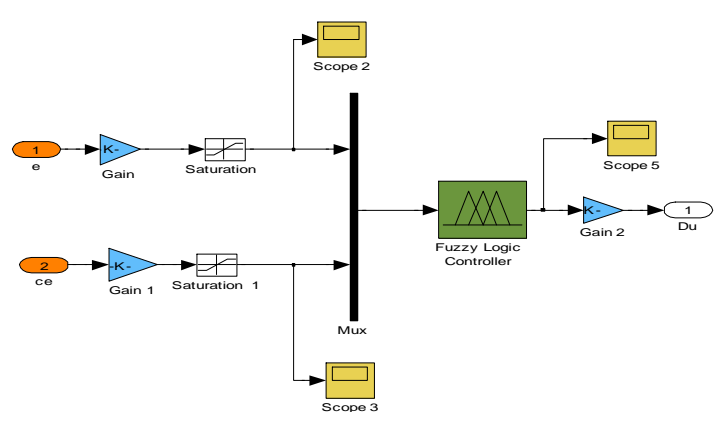

Fig .8 Simulink block diagram of FLC.

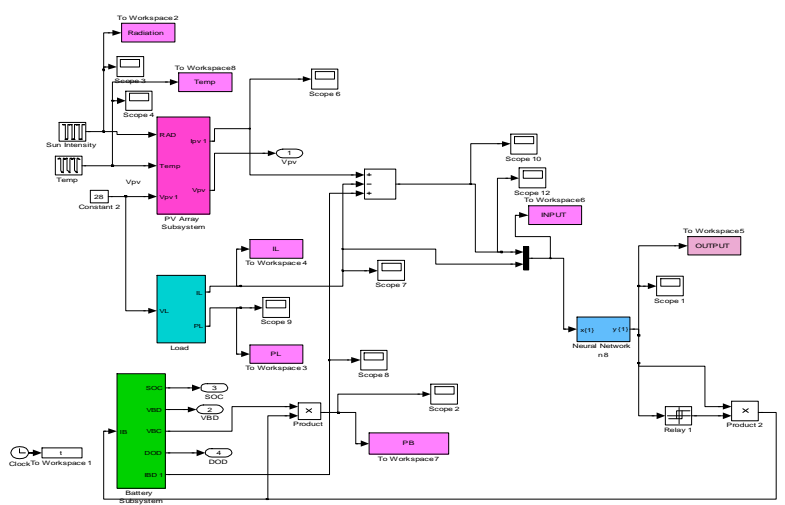

Fig.9 Simulink block diagram of the spacecraft power system with NNC. 

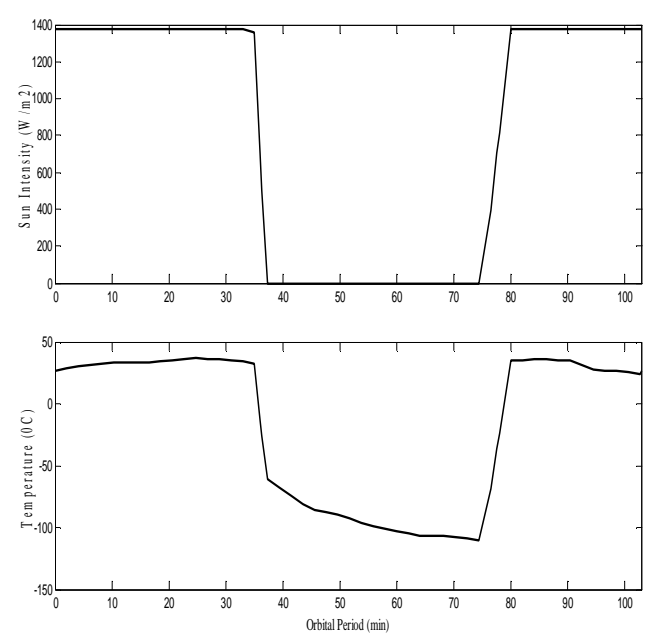

Fig. 10. Solar insolation and the temperature profile

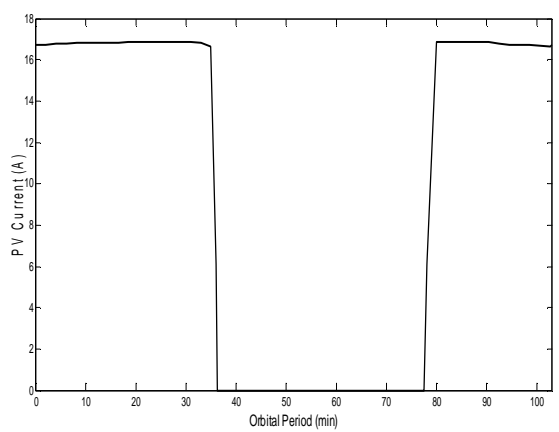

Fig. 11. The typical PV current
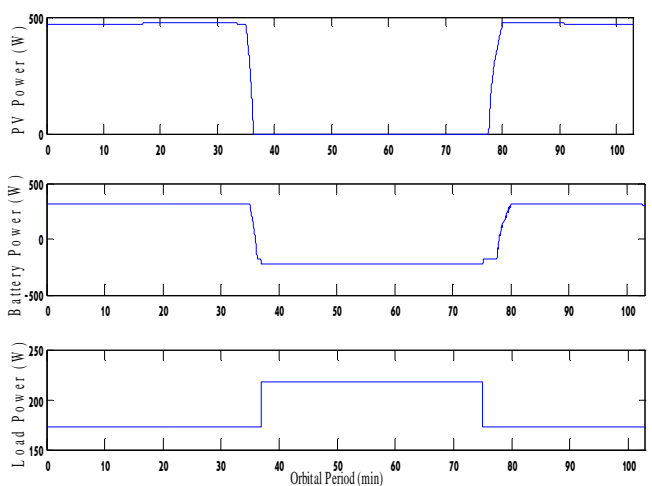

Fig. 12. The PV generated power, battery power, and Load Power using FLC.

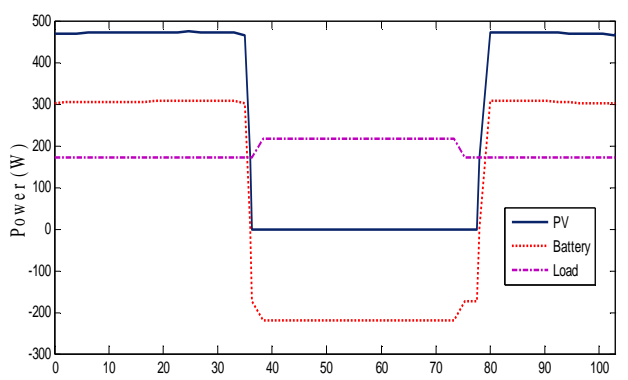

Fig. 13. The PV, battery, and load power profile using NNC.
The output generation of the control using either the FLC or the NNC is illustrated in Fig. 14. Also this figure shows the reference load, which is the total spacecraft load. Fig. 15 depicts zoomed region for the generated power using FLC. The FLC settling time is nearly 0.25 minuite to generate power while NNC takes approximately zero time to generate the required power. The error between the generation and load resulting with NNC \& FLC is shown in Fig. 16. It is cleared that the error resulting with FLC less than NNC during the orbital period while FLC doesn't generate the required power during the sun period. The NNC has a better response and accuracy than FLC for the following reasons: NNC is fed by various inputs which makes NNC more accurate, NNC satisfies the load power requirements, FLC settling time is larger than NNC, and NNC takes minimum time to simulate the whole system comparing to FLC.

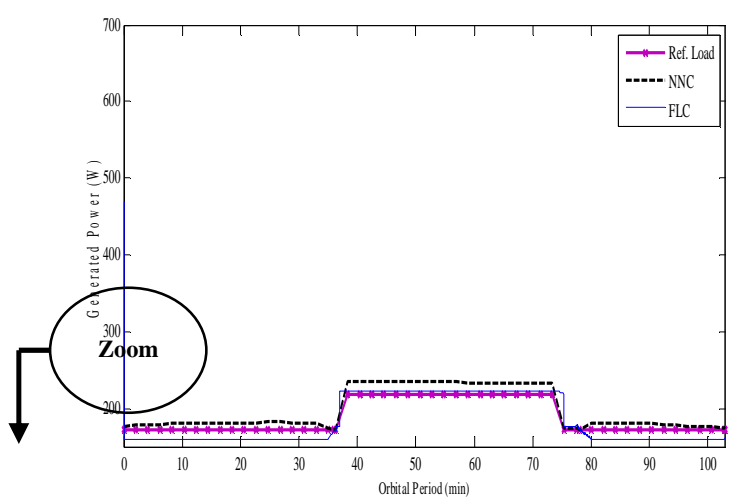

Fig. 14 Generated power with NNC and FLC over the orbital period.

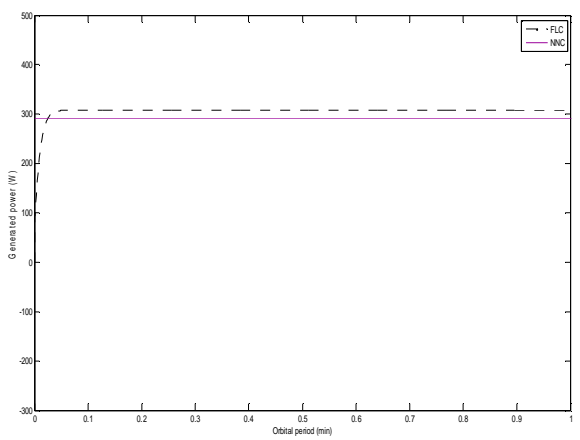

Fig. 15. Generated power with FLC \& NNC over the orbital period.

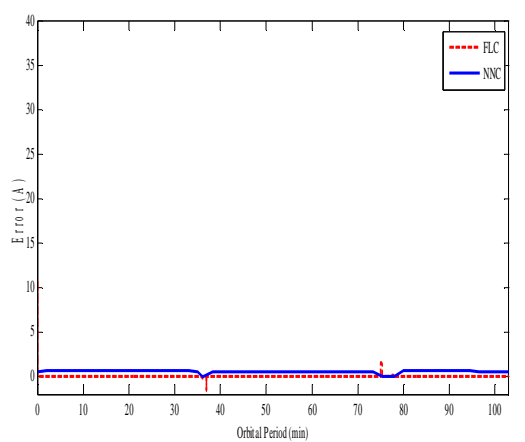

Fig. 16. Error resulting with FLC and NNC 


\section{Conclusion}

Artificial intelligence (AI) techniques are becoming useful as alternate approaches to conventional techniques or as components of integrated systems. The mathematical modeling and simulation of the spacecraft power system were carried out. FLC and NNC techniques are proposed to control spacecraft subsystems. This paper has presented a comparison of the NNC and FLC.

Off line training for the proposed NNC was applied. An ANN is trained using a back propagation with Levenberg-Marquardt algorithm. The best validation performance is obtained for mean square error is equal to $9.9962 \times 10^{-11}$ at epoch 637 . The regression between the network output and the corresponding target is equal to $100 \%$ which means a high accuracy. NNC architecture gives satisfactory results with small number of neurons, hence better in terms of memory and time are required for NNC implementation. The results indicate that the proposed control unit using ANN can be successfully used for controlling the spacecraft power system in low earth orbit (LEO) than FLC. Therefore, this technique is going to be a very useful tool for the interested designers in space field.

\section{References}

[1] Peter Fortescue, John Stark, and Graham Swinerd, "Spacecraft Systems Engineering", John Wiley \& Sons Ltd. , England, 2003.

[2] James A. Freeman and David M. Skapura, "Neural Networks Algorithms, Applications, And Programming Techniques", Addison-Wesley Publishing Company, Inc., Paris, 1991.

[3] Leocadio Hontoria, Jorge Aguilera, Florencia Almonacid, Gustavo Nofuentes and Pedro Zufiri, "Artificial Neural Networks Applied in PV Systems and Solar Radiation", Artificial Intelligence in Energy and Renewable, Nova Science Publishers, Inc. , 2006.

[4] Wiley J. Larson, and James R. Wertz, "Spacecraft Mission Analysis and Design", Micrcosm Press, Elo, Segrund, California, 2008.

[5] Sung-Soo Jang, and Jaeho Choi," Energy balance analysis of small satellite in Low Earth Orbit (LEO)," Proc. of $2^{\text {nd }}$ IEEE International Conference on Power and Energy (PECon 08), Johor Baharu, Malaysia. PP. 967-971, 2008.

[6] Kevin M. Passino and Stephen Yurkovich, "Fuzzy Control", Addison Wesley Longman, Inc. , 1998.

[7] Radu-Emil Precup, and Hans Hellendoorn, "A Survey on Industrial Applications of Fuzzy Control", Computers in Industry journal, 2010, under puplished.

[8] Kalogirou SA, "Artificial Intelligence in energy and renewable energy Systems", Nova Publisher, 2007.

[9] Ali Al-Alawi, Saleh M Al-Alawi, and Syed M Islam, "Predictive Control of an Integrated PV-diesel Water and Power Supply System Using an Artificial Neural Network," Renewable Energy , vol. 32, pp. 1426-1439, 2007.

[10] B. Chuco Paucar, J.L. Roel Ortiz, K.S. Collazos L., L.C.Leite, and J.O.P Pinto, "Power Operation Optimization of Photovoltaic Stand Alone System with Variable Loads Using Fuzzy Voltage Estimator and Neural Network Controller,” IEEE Power Tech. , 2007.
[11] Adel Mellita*, Mohamed Benghanemb, "Sizing of Standalone Photovoltaic Systems Using Neural Network Adaptive Model", Desalination Journal,Vol. 209, PP. 6472, 2007.

[12] S. Lalouni, D. Rekioua, T. Rekioua and E. Matagne, "Fuzzy Logic Control of Stand-Alone Photovoltaic System with Battery Storage", Journal of Power Sources, Vol. 193, PP. 899-907, 2009.

[13] Ch. Ben Salah, M. Chaaben, M. Ben Ammar, "Multicriteria Fuzzy Algorithm for Energy Management of a Domestic Photovoltaic Panel", Renewable Energy Vol. 33, PP. $993-1001,2008$.

[14] G. Colombo, U. Grasselli, A. De Luca, A. Spizzichino, and S. Falzinis, "Satellite Power System Simulation", Acta Astronautica, Vol. 40, No. I, PP. 4149, 1997. 